\title{
Reactor fuel fraction information on the antineutrino anomaly
}

\author{
C. Giunti, ${ }^{a}$ X.P. Ji, ${ }^{b}$ M. Laveder, ${ }^{c}$ Y.F. Li ${ }^{d, e}$ and B.R. Littlejohn ${ }^{f}$ \\ ${ }^{a}$ INFN, Sezione di Torino, \\ Via P. Giuria 1, I-10125 Torino, Italy \\ ${ }^{b}$ Department of Engineering Physics, Tsinghua University, \\ Qinghuayuan 1, 100084 Beijing, China \\ ${ }^{c}$ Dipartimento di Fisica e Astronomia "G. Galilei", Università di Padova, \\ and INFN, Sezione di Padova, \\ Via F. Marzolo 8, I-35131 Padova, Italy \\ ${ }^{d}$ Institute of High Energy Physics, Chinese Academy of Sciences, \\ Yuquan Lu 19B, 100049 Beijing, China \\ e School of Physical Sciences, University of Chinese Academy of Sciences, \\ Yuquan Lu 19B, 100049 Beijing, China \\ ${ }^{f}$ Physics Department, Illinois Institute of Technology, \\ 3105 S. Federal St, Chicago IL 60616, U.S.A. \\ E-mail: giunti@to.infn.it, jixp@mail.tsinghua.edu.cn, \\ laveder@pd.infn.it, liyufeng@ihep.ac.cn, blittlej@iit.edu
}

ABSTRACT: We analyzed the evolution data of the Daya Bay reactor neutrino experiment in terms of short-baseline active-sterile neutrino oscillations taking into account the theoretical uncertainties of the reactor antineutrino fluxes. We found that oscillations are disfavored at $2.6 \sigma$ with respect to a suppression of the ${ }^{235} \mathrm{U}$ reactor antineutrino flux and at $2.5 \sigma$ with respect to variations of the ${ }^{235} \mathrm{U}$ and ${ }^{239} \mathrm{Pu}$ fluxes. On the other hand, the analysis of the rates of the short-baseline reactor neutrino experiments favor active-sterile neutrino oscillations and disfavor the suppression of the ${ }^{235} \mathrm{U}$ flux at $3.1 \sigma$ and variations of the ${ }^{235} \mathrm{U}$ and ${ }^{239} \mathrm{Pu}$ fluxes at $2.8 \sigma$. We also found that both the Daya Bay evolution data and the global rate data are well-fitted with composite hypotheses including variations of the ${ }^{235} \mathrm{U}$ or ${ }^{239} \mathrm{Pu}$ fluxes in addition to active-sterile neutrino oscillations. A combined analysis of the Daya Bay evolution data and the global rate data shows a slight preference for oscillations with respect to variations of the ${ }^{235} \mathrm{U}$ and ${ }^{239} \mathrm{Pu}$ fluxes. However, the best fits of the combined data are given by the composite models, with a preference for the model with an enhancement of the ${ }^{239} \mathrm{Pu}$ flux and relatively large oscillations.

Keywords: Neutrino Physics, Beyond Standard Model

ARXIV EPRINT: 1708.01133 


\section{Contents}

1 Introduction 1

2 Daya Bay evolution 5

3 Previous reactor rates 10

4 Combined analysis $\quad 13$

5 Conclusions $\quad 15$

\section{Introduction}

Nuclear reactors are copious sources of electron antineutrinos $\left(\bar{\nu}_{e}\right)$, which have been detected by many experiments starting from the historical Reines and Cowan experiment in 1953-56 (see the reviews in refs. [1-3]). The electron antineutrinos are produced by $\beta$ decays of the neutron-rich nuclei generated by the fissions of the four fissionable isotopes ${ }^{235} \mathrm{U},{ }^{238} \mathrm{U},{ }^{239} \mathrm{Pu}$, and ${ }^{241} \mathrm{Pu}$. The total $\bar{\nu}_{e}$ flux emitted by each reactor is the sum of the fluxes generated by the four fissionable isotopes weighted by the effective fuel fractions which are monitored in time by the reactor managers.

The time evolution of nuclear reactors is divided in cycles of length that can go from about a month to one or two years. At the beginning of each cycle all or part of the fuel is replaced with fresh fuel, which is typically composed by uranium enriched with the fissile isotope ${ }^{235} \mathrm{U}$ with respect to the natural abundance, which is about $99.3 \%$ of ${ }^{238} \mathrm{U}$ and $0.7 \%$ of ${ }^{235} \mathrm{U}$. At the start of each reactor cycle the main contribution to the $\bar{\nu}_{e}$ flux comes from the fissions of ${ }^{235} \mathrm{U}$, with a small contribution of the fissionable isotope ${ }^{238} \mathrm{U}$. The neutron flux produced by the fissions generate mainly ${ }^{239} \mathrm{Pu}$ and in smaller quantity ${ }^{241} \mathrm{Pu}$. Hence, as ${ }^{235} \mathrm{U}$ is consumed during a cycle, the ${ }^{235} \mathrm{U}$ contribution to the $\bar{\nu}_{e}$ flux decreases and the contributions ${ }^{239} \mathrm{Pu}$ and ${ }^{241} \mathrm{Pu}$ increase, with a dominance of the ${ }^{239} \mathrm{Pu}$ contribution, which can be comparable with the ${ }^{235} \mathrm{U}$ contribution towards the end of each cycle.

For our discussion it is useful to distinguish two types of nuclear reactors: research reactors and power reactors. In research reactors the fresh fuel is made of almost pure ${ }^{235} \mathrm{U}$ and the cycles are short and give $\bar{\nu}_{e}$ fluxes which are practically due only to ${ }^{235} \mathrm{U}$. In most commercial power reactors the fresh fuel is made of uranium enriched by some percent of ${ }^{235} \mathrm{U}$ and the cycles are typically between one and two years. Therefore, the time evolution of the fuel composition must be taken into account in the calculation of the $\bar{\nu}_{e}$ flux of power reactors.

The $\bar{\nu}_{e}$ fluxes generated by the four fissionable isotopes have been calculated most recently in $2011[4,5]$, where they were found to be a few percent larger than in previous 
calculations [6-8]. The resulting predictions for the rates of $\bar{\nu}_{e}$-induced events measured in several neutrino experiments are smaller than the experimental rates. The average deficit of about $5 \%$ is the so-called "reactor antineutrino anomaly" [9], which can be due to two causes: a) some mistake in the $\bar{\nu}_{e}$ flux calculations and/or b) the disappearance of $\bar{\nu}_{e}$ during their propagation from the reactor to the detector. This disappearance is most likely due to active-sterile neutrino oscillations (see the review in ref. [10]).

The Daya Bay collaboration presented recently [11] the results of the measurement of the correlation between the reactor fuel evolution and the changes in the antineutrino detection rate in the Daya Bay experiment, which detects $\bar{\nu}_{e}$ 's produced by two complexes of power reactors at distances of about $360 \mathrm{~m}$ and $500 \mathrm{~m}$. The $\bar{\nu}_{e}$ detection rate is quantified by the cross section per fission $\sigma_{f}$, given by

$$
\sigma_{f}=\sum_{i} F_{i} \sigma_{f, i}
$$

where $F_{i}$ and $\sigma_{f, i}$ are the effective fission fractions and the cross sections per fission of the four fissionable isotopes ${ }^{235} \mathrm{U},{ }^{238} \mathrm{U},{ }^{239} \mathrm{Pu},{ }^{241} \mathrm{Pu}$, denoted, respectively, with the label $i=235,238,239,241$.

The Daya Bay collaboration presented in figure 2 of ref. [11] the values of $\sigma_{f}$ for eight values of the effective ${ }^{239} \mathrm{Pu}$ fission fraction $F_{239}$. They fitted these data allowing variations of the two main cross sections per fission $\sigma_{f, 235}$ and $\sigma_{f, 239}$, with the assumption that $\sigma_{f, 238}$ and $\sigma_{f, 241}$ have the Saclay+Huber theoretical values $[4,5,9]$ with enlarged $10 \%$ uncertainties. They also compared the best-fit of this analysis with the best-fit obtained under the hypothesis of active-sterile neutrino oscillations, which predicts the same suppression for the four cross sections per fission with respect to their theoretical value. They obtained $\Delta \chi^{2} / \mathrm{NDF}=7.9 / 1$, corresponding to a $p$-value of $0.49 \%$, which disfavors the active-sterile oscillations hypothesis by $2.8 \sigma$. In this calculation the uncertainties of the theoretical calculation of the four cross sections per fission were not taken into account.

In this paper we present the results of analyses of the Daya Bay evolution data [11] with least-squares functions that take into account explicitly the uncertainties of the theoretical calculation of the four cross sections per fission. Moreover, we consider additional models with independent variations of the ${ }^{235} \mathrm{U}$ and ${ }^{239} \mathrm{Pu}$ fluxes with and without active-sterile neutrino oscillations, and we extend the analysis taking into account also the information on the cross sections per fission of all the other reactor antineutrino experiments which have different fuel fractions. We also perform proper statistical comparisons of the non-nested models under consideration through Monte Carlo estimations of the $p$-values.

Given a set of data labeled with the index $a$ on the cross section per fission for different values of the fuel fractions, we write the theoretical predictions as

$$
\sigma_{f, a}^{\mathrm{th}}=\sum_{i} F_{i}^{a} r_{i} \sigma_{f, i}^{\mathrm{SH}}
$$

where $i=235,238,239,241$ and $\sigma_{f, i}^{\mathrm{SH}}$ are the Saclay+Huber cross sections per fission. The coefficients $r_{i}$ are introduced in order to take into account the uncertainties of the 
Saclay+Huber cross sections per fission or to study independent variations of the antineutrino fluxes from the four fissionable isotopes with respect to the Saclay+Huber theoretical values $[4,5,9]$.

We consider the following models:

235 A variation of the cross section per fission of the antineutrino flux from ${ }^{235} \mathrm{U}$ only. In this case, we analyze the data with the least-squares statistic

$$
\begin{aligned}
\chi^{2}= & \sum_{a, b}\left(\sigma_{f, a}^{\mathrm{th}}-\sigma_{f, a}^{\exp }\right)\left(V_{\exp }^{-1}\right)_{a b}\left(\sigma_{f, b}^{\mathrm{th}}-\sigma_{f, b}^{\exp }\right) \\
& +\sum_{i, j=238,239,241}\left(r_{i}-1\right)\left(V_{\mathrm{SH}}^{-1}\right)_{i j}\left(r_{j}-1\right),
\end{aligned}
$$

where $\sigma_{f, a}^{\exp }$ are the measured cross sections per fission, $V_{\exp }$ is the experimental covariance matrix, and $V_{\mathrm{SH}}$ is the covariance matrix of the fractional uncertainties of the Saclay-Huber theoretical calculation of the antineutrino fluxes from the four fissionable isotopes (given in table 3 of ref. [12]).

In this analysis there is only one parameter determined by the fit: $r_{235}$. The parameters $r_{238}, r_{239}$, and $r_{241}$ are nuisance parameters.

$\mathbf{2 3 5}+\mathbf{2 3 9}$ Independent variations of the cross sections per fission of the antineutrino fluxes from ${ }^{235} \mathrm{U}$ and ${ }^{239} \mathrm{Pu}$.

In this case, we analyze the data with the least-squares statistic

$$
\begin{aligned}
\chi^{2}= & \sum_{a, b}\left(\sigma_{f, a}^{\mathrm{th}}-\sigma_{f, a}^{\exp }\right)\left(V_{\exp }^{-1}\right)_{a b}\left(\sigma_{f, b}^{\mathrm{th}}-\sigma_{f, b}^{\exp }\right) \\
& +\sum_{i, j=238,241}\left(r_{i}-1\right)\left(V_{\mathrm{SH}}^{-1}\right)_{i j}\left(r_{j}-1\right) .
\end{aligned}
$$

In this analysis there are two parameters determined by the fit: $r_{235}$ and $r_{239}$. The parameters $r_{238}$ and $r_{241}$ are nuisance parameters.

OSC Active-sterile neutrino oscillations, in which the measured cross sections per fission are suppressed with respect to the theoretical cross sections per fission $\sigma_{f, a}^{\text {th }}$ by the survival probability $P_{e e}$ which is independent of the ${ }^{239} \mathrm{Pu}$ fraction $F_{239}$.

In this case, we analyze the data with the least-squares statistic

$$
\begin{aligned}
\chi^{2}= & \sum_{a, b}\left(P_{e e} \sigma_{f, a}^{\mathrm{th}}-\sigma_{f, a}^{\exp }\right)\left(V_{\exp }^{-1}\right)_{a b}\left(P_{e e} \sigma_{f, b}^{\mathrm{th}}-\sigma_{f, b}^{\exp }\right) \\
& +\sum_{i, j}\left(r_{i}-1\right)\left(V_{\mathrm{SH}}^{-1}\right)_{i j}\left(r_{j}-1\right) .
\end{aligned}
$$

In the analysis of the Daya Bay evolution data there is only one parameter determined by the fit: $P_{e e}$. The parameters $r_{235}, r_{238}, r_{239}$, and $r_{241}$ are nuisance parameters. In the analysis of the other reactor antineutrino data we take into account that $P_{e e}$ 
depends on the neutrino mixing parameters $\Delta m_{41}^{2}$ and $\sin ^{2} 2 \vartheta_{e e}$ in the simplest $3+1$ active-sterile neutrino mixing model (see ref. [10]). Hence, in this case there are two parameters determined by the fit: $\Delta m_{41}^{2}$ and $\sin ^{2} 2 \vartheta_{e e}$.

235+OSC A variation of the cross section per fission of the antineutrino flux from ${ }^{235} \mathrm{U}$ and active-sterile neutrino oscillations with a survival probability $P_{e e}$ as in the OSC model.

In this case, we analyze the data with the least-squares statistic

$$
\begin{aligned}
\chi^{2}= & \sum_{a, b}\left(P_{e e} \sigma_{f, a}^{\mathrm{th}}-\sigma_{f, a}^{\exp }\right)\left(V_{\exp }^{-1}\right)_{a b}\left(P_{e e} \sigma_{f, b}^{\mathrm{th}}-\sigma_{f, b}^{\exp }\right) \\
& +\sum_{i, j=238,239,241}\left(r_{i}-1\right)\left(V_{\mathrm{SH}}^{-1}\right)_{i j}\left(r_{j}-1\right) .
\end{aligned}
$$

In the analysis of the Daya Bay evolution data there are two parameters determined by the fit: $r_{235}$ and $P_{e e}$. The parameters $r_{238}, r_{239}$ and $r_{241}$ are nuisance parameters. In the analysis of the other reactor antineutrino data we take into account that $P_{e e}$ depends on $\Delta m_{41}^{2}$ and $\sin ^{2} 2 \vartheta_{e e}$ as in the OSC model. Therefore, in this case there are three parameters determined by the fit: $r_{235}, \Delta m_{41}^{2}$, and $\sin ^{2} 2 \vartheta_{e e}$.

239+OSC This model is similar to the $235+$ OSC model, with ${ }^{235} \mathrm{U} \leftrightarrows{ }^{239} \mathrm{Pu}$. The number of parameters determined by the fit is two in the analysis of the Daya Bay evolution data $\left(r_{239}\right.$ and $\left.P_{e e}\right)$ and three in the analysis of the other reactor antineutrino data $\left(r_{239}, \Delta m_{41}^{2}\right.$, and $\left.\sin ^{2} 2 \vartheta_{e e}\right)$.

Note that:

1. In all the models we constrained the values of $\sigma_{f, 238}$ and $\sigma_{f, 241}$ around the corresponding theoretical values with the theoretical uncertainties. In principle it would be interesting to consider also $\sigma_{f, 238}$ and $\sigma_{f, 241}$ as free parameters to be determined by the fit of the data. However, this is not possible in practice because the data do not constrain them in a sufficient way. Indeed, also the Daya Bay collaboration [11] constrained $\sigma_{f, 238}$ and $\sigma_{f, 241}$ around the theoretical values, albeit with an arbitrary $10 \%$ uncertainty. We adopt the theoretical uncertainties because they are well defined and motivated, and it is possible that the corresponding predictions are correct, whereas those of $\sigma_{f, 235}$ and $\sigma_{f, 239}$ are not.

2. We did not consider the case $\mathbf{2 3 5}+\mathbf{2 3 9}+\mathbf{O S C}$, because this model is not constrained by the data. This is due to the fact that arbitrarily large values of $\sigma_{f, 235}$ and $\sigma_{f, 239}$ can be counterbalanced by an arbitrary small $P_{e e}$.

The plan of the paper is as follows: in section 2 we analyze the Daya Bay evolution data, in section 3 we analyze the reactor antineutrino data which were available before the release of the Daya Bay fuel evolution data in ref. [11], in section 4 we perform the combined analysis, and in section 5 we draw our conclusions. 


\begin{tabular}{|cccccc|}
\hline & 235 & $235+239$ & OSC & $235+$ OSC & $239+$ OSC \\
\hline$\chi_{\min }^{2}$ & 3.8 & 3.6 & 9.5 & 3.6 & 3.8 \\
$\mathrm{NDF}$ & 7 & 6 & 7 & 6 & 6 \\
$\mathrm{GoF}$ & $80 \%$ & $73 \%$ & $22 \%$ & $72 \%$ & $71 \%$ \\
$P_{e e}$ & - & - & 0.942 & 0.984 & 0.928 \\
$r_{235}$ & 0.927 & 0.922 & - & 0.937 & - \\
$r_{239}$ & - & 0.974 & - & - & 1.094 \\
\hline
\end{tabular}

Table 1. Fits of the Daya Bay evolution data [11].

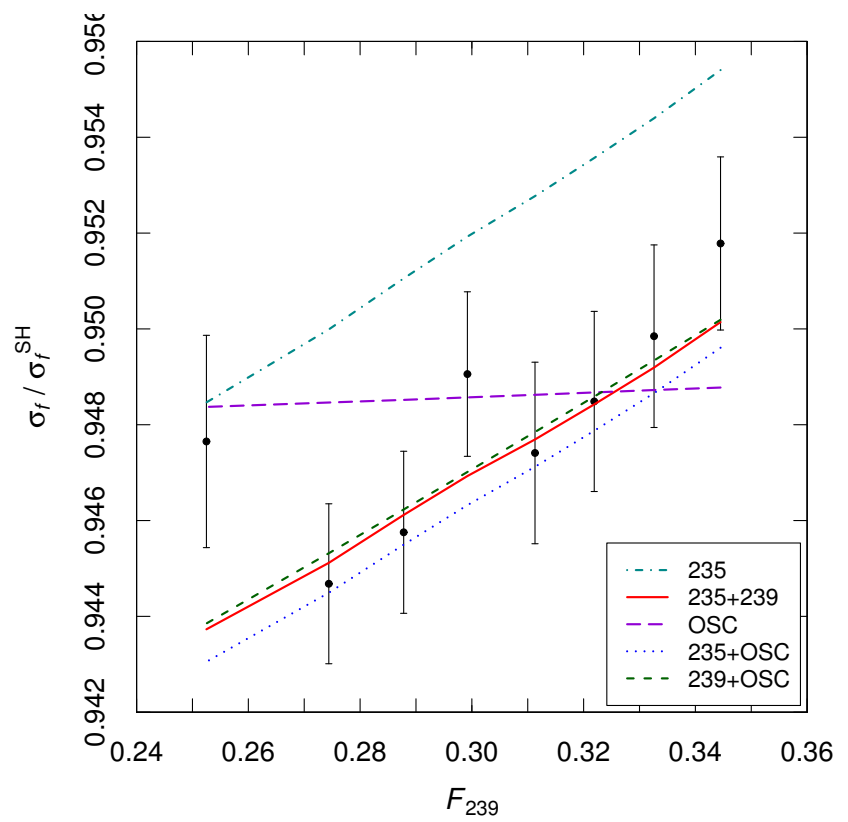

Figure 1. Fits of the Daya Bay evolution data [11] normalized to the Saclay-Huber theoretical predictions $[4,5,9]$. The error bars show only the uncorrelated statistical uncertainties.

\section{Daya Bay evolution}

The results of the different fits of the Daya Bay evolution data are given in table 1 where we list the values of the minimum $\chi^{2}$, the number of degrees of freedom and the goodness-of-fit. In table 1 we also list the best-fit values of the fitted parameters.

Figure 1 shows the comparison of the different fits with the Daya Bay evolution data normalized to the Saclay-Huber theoretical cross sections per fission $[4,5,9]$. Note that the Daya Bay evolution data have the following two important features:

F1 A suppression of $\sigma_{f}$ with respect to $\sigma_{f}^{\mathrm{SH}}$ in agreement with the reactor antineutrino anomaly. This feature can be fitted with at least one of the $r_{i}$ and $P_{e e}$ smaller than one (if the others are equal to one). 
F2 An increase of $\sigma_{f} / \sigma_{f}^{\mathrm{SH}}$ with $F_{239}$. This feature can be fitted if

$$
\frac{d}{d F_{239}} \frac{\sigma_{f, a}^{\mathrm{th}}}{\sigma_{f, a}^{\mathrm{SH}}}>0,
$$

where

$$
\sigma_{f, a}^{\mathrm{SH}}=\sum_{i} F_{i}^{a} \sigma_{f, i}^{\mathrm{SH}} .
$$

The inequality (2.1) is satisfied for

$$
\sum_{i} \frac{d F_{i}^{a}}{d F_{239}} r_{i} \sigma_{f, i}^{\mathrm{SH}}>\frac{\sigma_{f, a}^{\mathrm{th}}}{\sigma_{f, a}^{\mathrm{SH}}} \frac{d \sigma_{f, a}^{\mathrm{SH}}}{d F_{239}},
$$

with

$$
\frac{d \sigma_{f, a}^{\mathrm{SH}}}{d F_{239}} \simeq-2.4<0 .
$$

From table 1 one can see that all the fits have acceptable goodness-of-fit, but the OSC fit corresponding to active-sterile oscillations has a goodness-of-fit which is significantly lower than the others, because it corresponds to a constant $\sigma_{f} / \sigma_{f}^{\mathrm{SH}}$ and cannot fit feature F2.

The results of our analysis agree with the conclusion of the Daya Bay collaboration [11] that the 235 model fits well the data and little is gained by allowing also the variation of $\sigma_{f, 239}$ in the $235+239$ model. The shift in figure 1 of the line corresponding to the 235 model with respect to an ideal line fitting the data by eye is allowed by the large correlated systematic uncertainties of the Daya Bay bins [11].

The excellent fit in the 235 model is due to the fact that it can fit the two features of the Daya Bay evolution data listed above. It can obviously fit feature F1 with $r_{235}<1$. It can also fit feature F2, because for $r_{235}<1$ and $r_{238}=r_{239}=r_{241}=1$ the condition $(2.3)$ becomes

$$
-\frac{d F_{235}^{a}}{d F_{239}}>-\frac{d \sigma_{f, a}^{\mathrm{SH}}}{d F_{239}} \frac{F_{235}^{a}}{\sigma_{f, a}^{\mathrm{SH}}} .
$$

This condition is satisfied, because numerically the left-hand side is about 1.30 and the right-hand side is between 0.20 and 0.24 .

Obviously, the $235+$ OSC model can provide a fit which is at least as good as the 235 model, with the additional possibility to improve the fit of feature F1 with $P_{e e}<1$.

It is maybe more surprising that also the 239+OSC model fits better than the 235 model for $r_{239}>1$. This can happen because the condition (2.3) for fitting feature F2 is always satisfied for $r_{239}>1$ and $r_{235}=r_{238}=r_{241}=1$. Then, a sufficiently small value of $P_{e e}<1$ allows us to fit feature F1 in spite of the increase of $\sigma_{f, a}^{\text {th }}$ due to $r_{239}>1$.

Nested models can be compared in the frequentist approach by calculating the $p$-value of the $\chi_{\min }^{2}$ difference, which has a $\chi^{2}$ distribution corresponding to the difference of the number of degrees of freedom of the two models. With this method we can compare only the nested models 235 and $235+$ OSC, because the $\chi^{2}$ in eq. (1.3) can be obtained from that in eq. (1.6) with the constraint $P_{e e}=1$. In this comparison, we have $\Delta \chi^{2}=0.2$ with 


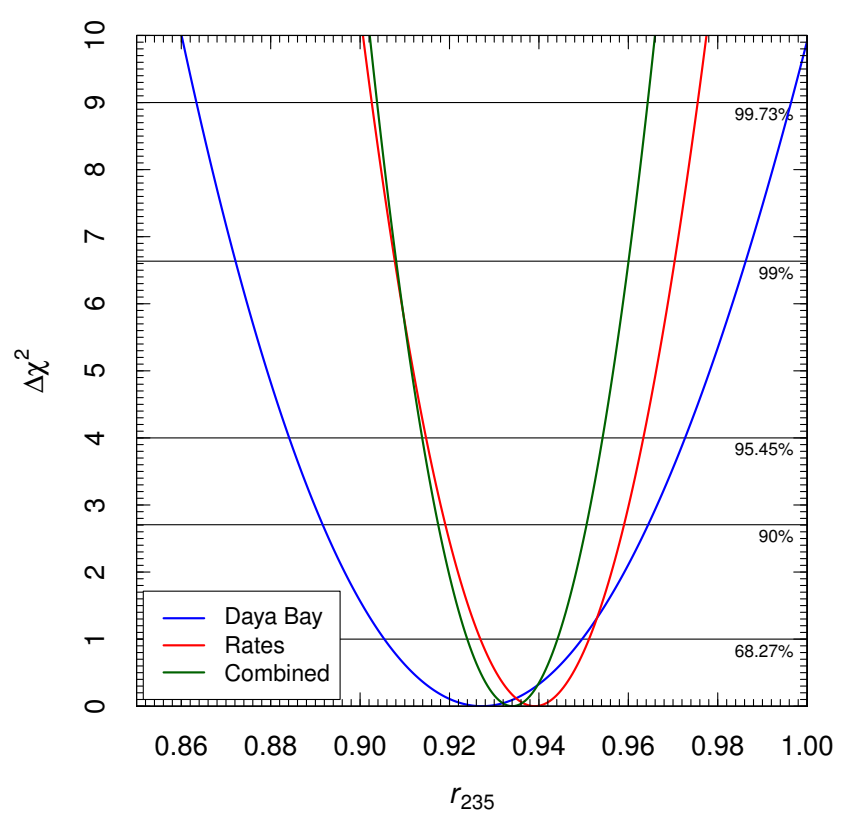

Figure 2. Marginal $\Delta \chi^{2}=\chi^{2}-\chi_{\min }^{2}$ for the factor $r_{235}$ obtained from the fit of the Daya Bay evolution data [11] (Daya Bay), from the fit of the reactor rates (Rates), and from the combined fit (Combined) with the 235 model.

one degree of freedom. Hence, the null hypothesis 235 cannot be rejected in favor of the alternative more complex hypothesis $235+$ OSC.

Also non-nested models can be compared considering the $\chi_{\min }^{2}$ difference, but one must calculate the $p$-value with a Monte Carlo. In this case one must consider as the null hypothesis the model which has the higher $\chi_{\min }^{2}$ and generate many sets of synthetic data assuming the null hypothesis. The fits of all the sets of synthetic data with the two models under consideration gives the distribution of the $\chi_{\min }^{2}$ difference from which one can calculate the $p$-value of the observed $\chi_{\min }^{2}$ difference.

We do not bother to consider the comparison of the 235 and $235+239$ models, since the small $\Delta \chi_{\min }^{2}=0.2$ cannot lead to the rejection of the null hypothesis 235 .

On the other hand, it is interesting to compare the OSC and 235 models which have $\Delta \chi_{\min }^{2}=5.7$. According to our Monte Carlo simulation, the $p$-value of the null hypothesis OSC is $0.85 \%$. Hence, the comparison of the OSC and 235 models disfavors the OSC model at the $2.6 \sigma$ level.

We also compared with a Monte Carlo the OSC and 235+239 models which have $\Delta \chi_{\min }^{2}=5.9$. We found that the null hypothesis OSC has a $p$-value of $1.3 \%$, which is larger than in the previous case because the $235+239$ model has one parameter more than the 235 model. Thus, in this case, the OSC model is disfavored at the $2.5 \sigma$ level, which is slightly less stringent than the $2.8 \sigma$ obtained by the Daya Bay collaboration [11] without considering the theoretical uncertainties.

Figures 2, 3, 4, 5, and 6 show the allowed regions of the fitted parameters in the 235, 235+239, OSC, $235+\mathrm{OSC}$, and 239+OSC models, respectively. In these figures, 


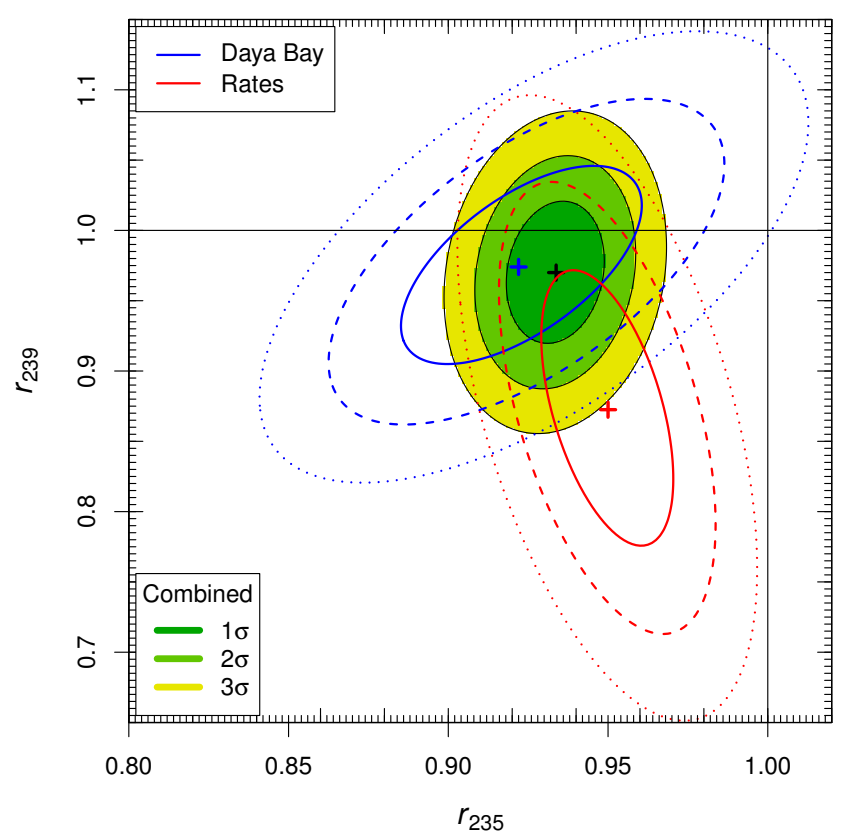

Figure 3. Allowed regions in the $r_{235}-r_{239}$ plane obtained from the fit of the Daya Bay evolution data [11] (Daya Bay), from the fit of the reactor rates (Rates), and from the combined fit (Combined) with the $235+239$ model. The best fit points are indicated by crosses. For the Daya Bay and Rates fits the $1 \sigma, 2 \sigma$, and $3 \sigma$ allowed regions are limited, respectively, by solid, dashed, and dotted lines.

the results of the fit of the Daya Bay evolution data are compared with those of the fit of the reactor rates discussed in section 3 and those of the combined fit discussed in section 4 .

From figure 2, one can see that assuming the 235 model, the fit of the Daya Bay evolution data gives

$$
r_{235}=0.927 \pm 0.022
$$

which determines the ${ }^{235} \mathrm{U}$ cross section per fission to be

$$
\sigma_{f, 235}=6.20 \pm 0.15
$$

In the case of the $235+239$ model, figure 3 show that the Daya Bay evolution data indicate a larger suppression of $\sigma_{f, 235}$ than $\sigma_{f, 239}$, in agreement with the results of the analysis of the Daya Bay collaboration [11]. We obtained

$$
\begin{aligned}
& r_{235}=0.922 \pm 0.025, \\
& r_{239}=0.974 \pm 0.046,
\end{aligned}
$$

which imply

$$
\begin{aligned}
& \sigma_{f, 235}=6.17 \pm 0.16, \\
& \sigma_{f, 239}=4.29 \pm 0.20 .
\end{aligned}
$$

These results are compatible with those obtained by the Daya Bay collaboration [11], taking into account of the different assumptions on the uncertainties of $\sigma_{f, 238}$ and $\sigma_{f, 241}$ 


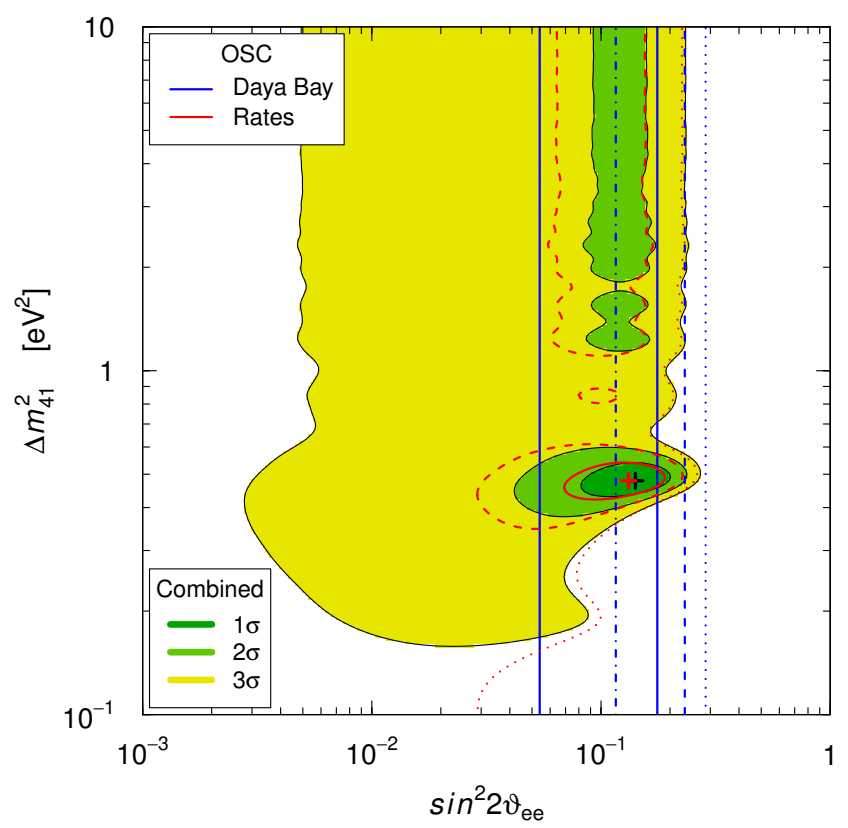

Figure 4. Allowed regions in the $\sin ^{2} 2 \vartheta_{e e}-\Delta m_{41}^{2}$ plane obtained from the fit of the Daya Bay evolution data [11] (Daya Bay), from the fit of the reactor rates (Rates), and from the combined fit (Combined) with the OSC model. The best fit points are indicated by crosses, except for the fit of the Daya Bay evolution data for which the best fit is the vertical dash-dotted line. For the Daya Bay and Rates fits the $1 \sigma, 2 \sigma$, and $3 \sigma$ allowed regions are limited, respectively, by solid, dashed, and dotted lines.

(10\% in the calculation of the Daya Bay collaboration and the Saclay+Huber theoretical values $[4,5,9] 8.15 \%$ and $2.60 \%$ in our calculation).

The vertical lines in figure 4 show the bounds on $\sin ^{2} 2 \vartheta_{e e}=2\left(1-P_{e e}\right)$ obtained in the OSC analysis of the Daya Bay evolution data, in which oscillations are averaged because of the large source-detector distance. One can see that

$$
\sin ^{2} 2 \vartheta_{e e}=0.12 \pm 0.06
$$

and there is no lower bound at $2 \sigma$, because oscillations are favored over the no-oscillation case only at the $1.9 \sigma$ level.

Figure 5 shows that the variation of $r_{235}$ in the $235+$ OSC model causes a shift of the allowed region for $\sin ^{2} 2 \vartheta_{e e}$ towards lower values with respect to figure 4 obtained with the OSC. In figure 5 there is no lower bound at $1 \sigma$, because oscillations are favored over the no-oscillation case only at $0.4 \sigma$. This is due to the preference for values of $r_{235}$ smaller than one, as shown by the best-fit value in table 1 .

On the other hand, in figure 6 corresponding to the 239+OSC there is a shift of the allowed region for $\sin ^{2} 2 \vartheta_{e e}$ towards larger values with respect to figure 4 obtained with the OSC, because $P_{e e}$ is smaller in order to compensate the increase of $\sigma_{f, a}^{\text {th }}$ due to $r_{239}>1$. 


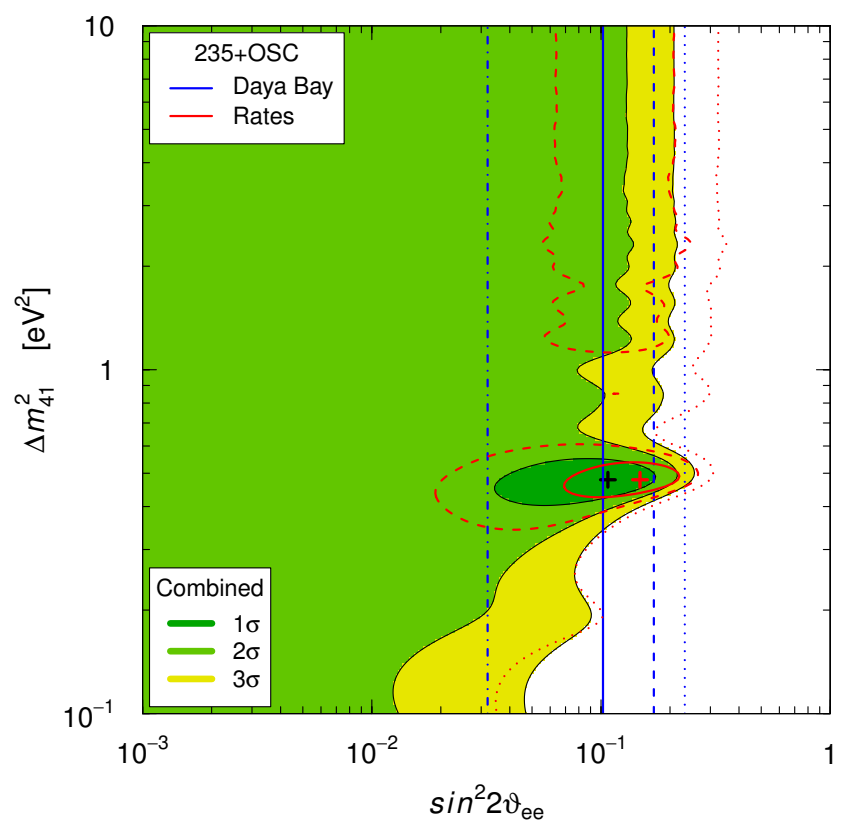

Figure 5. Allowed regions in the $\sin ^{2} 2 \vartheta_{e e}-\Delta m_{41}^{2}$ plane obtained from the fit of the Daya Bay evolution data [11] (Daya Bay), from the fit of the reactor rates (Rates), and from the combined fit (Combined) with the $235+$ OSC model. The best fit points are indicated by crosses, except for the fit of the Daya Bay evolution data for which the best fit is the vertical dash-dotted line. For the Daya Bay and Rates fits the $1 \sigma, 2 \sigma$, and $3 \sigma$ allowed regions are limited, respectively, by solid, dashed, and dotted lines.

\section{Previous reactor rates}

In this section we consider the reactor antineutrino data which were available before the release of the Daya Bay fuel evolution data in ref. [11]. We use the data listed in table 1 of ref. [12] of the following experiments: Bugey-4 [13], Rovno91 [14], Bugey-3 [15], Gosgen [16], ILL [17, 18], Krasnoyarsk87 [19], Krasnoyarsk94 [20, 21], Rovno88 [22], SRP [23], Nucifer [24], Chooz [25], Palo Verde [26], Daya Bay [27], RENO [28], and Double Chooz [29]. The Daya Bay data in ref. [27] are relative to the average Daya Bay fuel fractions for the corresponding detection time.

The results of the fits with the models described in section 1 are listed in table 2 and the fit of the data is illustrated in figures 7 and 8 .

From table 2 one can see that all the model have an excellent goodness-of-fit, but the models OSC, 235+OSC, and 239+OSC with active-sterile neutrino oscillations have a significantly lower value of $\chi_{\min }^{2}$. This is due to the different source-detector distances in the experiments. As one can see from figure 7, where the reactor data are ordered by increasing values of the source-detector distance $L$. One can see that active-sterile oscillations can fit better the data of the short-baseline experiments which have a source detector distance between about 10 and $100 \mathrm{~m}$. On the other hand, the poor fit of the data with the 235 model is explained by the lack of a trend figure 8 , where the reactor data are ordered by decreasing values of $F_{235}$. 


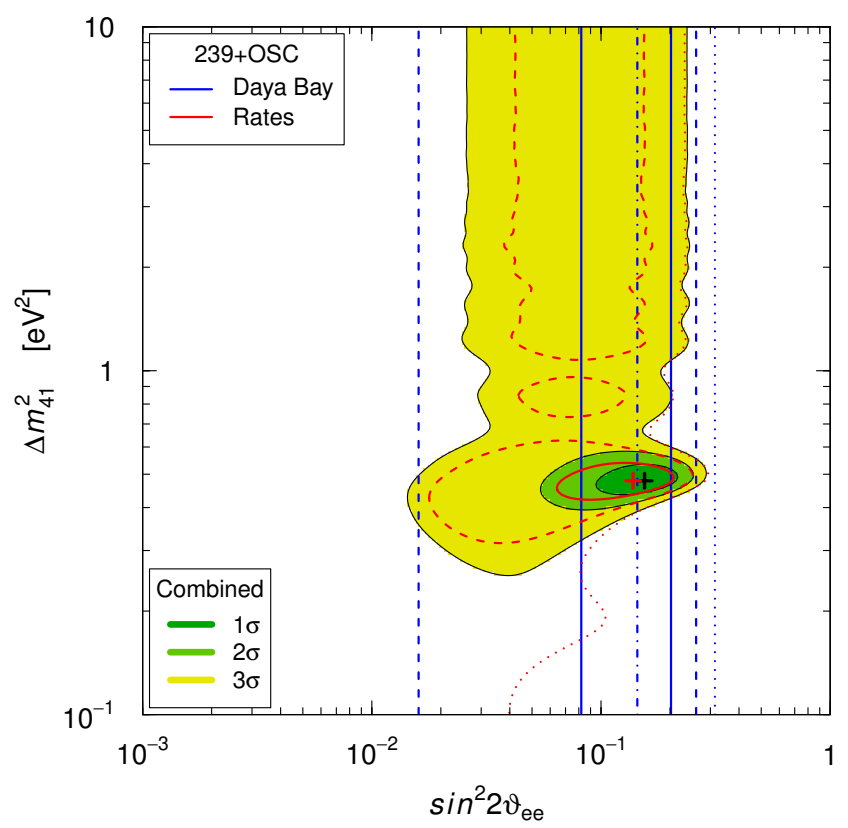

Figure 6. Allowed regions in the $\sin ^{2} 2 \vartheta_{e e}-\Delta m_{41}^{2}$ plane obtained from the fit of the Daya Bay evolution data [11] (Daya Bay), from the fit of the reactor rates (Rates), and from the combined fit (Combined) with the 239+OSC model. The best fit points are indicated by crosses, except for the fit of the Daya Bay evolution data for which the best fit is the vertical dash-dotted line. For the Daya Bay and Rates fits the $1 \sigma, 2 \sigma$, and $3 \sigma$ allowed regions are limited, respectively, by solid, dashed, and dotted lines.

\begin{tabular}{|cccccc|}
\hline & 235 & $235+239$ & OSC & $235+$ OSC & $239+\mathrm{OSC}$ \\
\hline$\chi_{\min }^{2}$ & 20.7 & 17.7 & 12.8 & 12.6 & 12.7 \\
$\mathrm{NDF}$ & 25 & 24 & 24 & 23 & 23 \\
$\mathrm{GoF}$ & $71 \%$ & $82 \%$ & $100 \%$ & $100 \%$ & $100 \%$ \\
$\Delta m_{41}^{2}$ & - & - & 0.48 & 0.48 & 0.48 \\
$\sin ^{2} 2 \vartheta_{e e}$ & - & - & 0.13 & 0.15 & 0.14 \\
$r_{235}$ & 0.939 & 0.950 & - & 1.025 & - \\
$r_{239}$ & - & 0.873 & - & - & 1.036 \\
\hline
\end{tabular}

Table 2. Fits of the reactor rates in table 1 of ref. [12].

The comparison of the nested models 235 and $235+\mathrm{OSC}$ give $\Delta \chi_{\min }^{2}=8.1$ with two degrees of freedom. Hence, the $p$-value of the null hypothesis 235 is $1.7 \%$ and it can be rejected in favor of the introduction of active-sterile neutrino oscillations at $2.4 \sigma$. As a check, with a Monte Carlo simulation we obtained a $p$-value of $1.3 \%$, which corresponds to $2.5 \sigma$.

The 235 and OSC models have $\Delta \chi_{\min }^{2}=7.9$ and our Monte Carlo comparison disfavors the null hypothesis 235 at $3.1 \sigma$.

The $235+239$ and OSC models have $\Delta \chi_{\min }^{2}=4.9$ and our Monte Carlo comparison disfavors the null hypothesis $235+239$ at $2.8 \sigma$. 

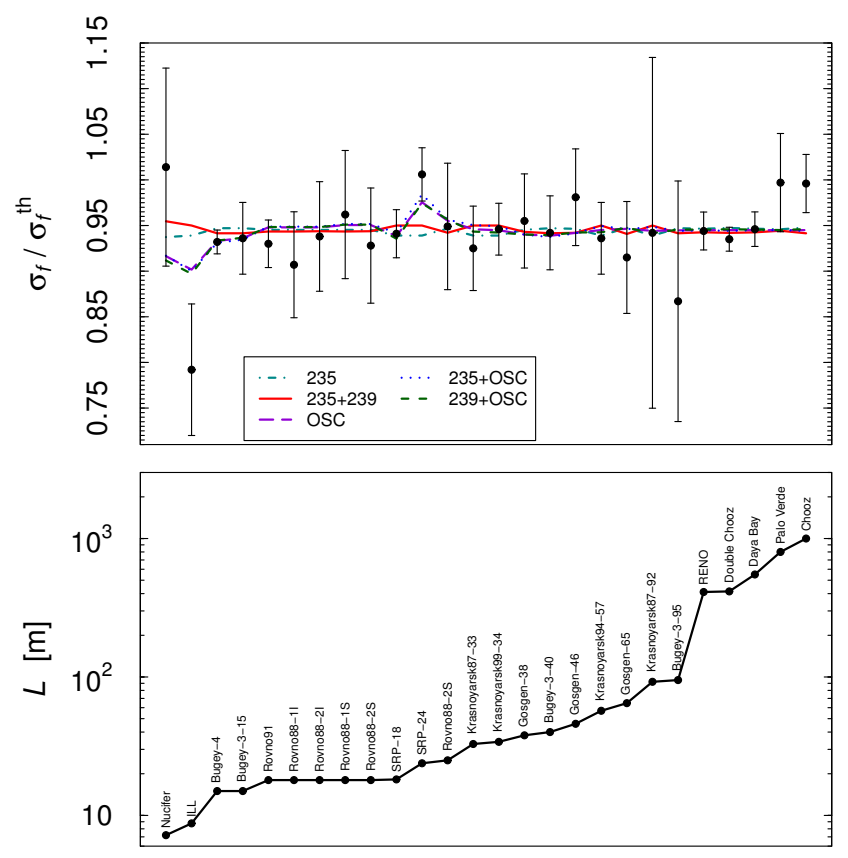

Figure 7. The top panels show the fits of the reactor rates in table 1 of ref. [12]. The data are ordered by increasing values of the source-detector distance $L$, shown in the bottom panel. The error bars show to the experimental uncertainties.

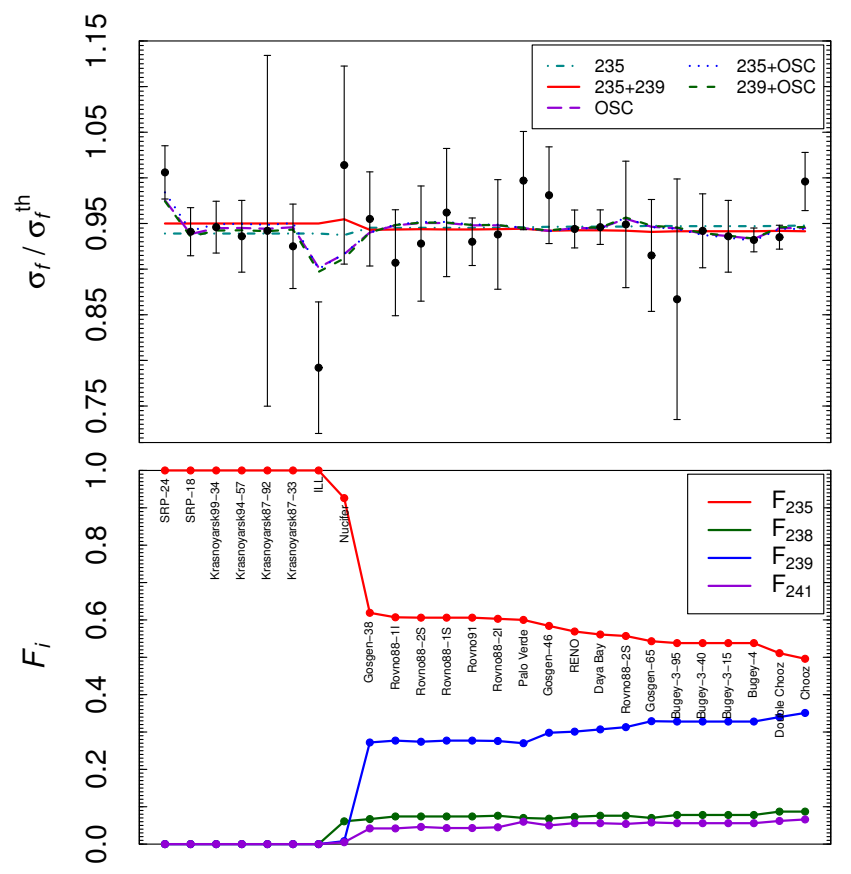

Figure 8. The top panels show the fits of the reactor rates in table 1 of ref. [12]. The data are ordered by decreasing values of $F_{235}$, shown in the bottom panel. The error bars show to the experimental uncertainties. 
Figure 2 shows the marginal $\Delta \chi^{2}=\chi^{2}-\chi_{\min }^{2}$ for the factor $r_{235}$ obtained from the fit of the reactor rates in the 235 model. The result is

$$
r_{235}=0.939 \pm 0.012
$$

which gives

$$
\sigma_{f, 235}=6.28 \pm 0.08 \text {. }
$$

This is a determination of $\sigma_{f, 235}$ with smaller uncertainty than that obtained in eq. (2.7) from the Daya Bay evolution data.

Figure 3 shows that in the case of the $235+239$ model the determination of $r_{235}$ and $r_{239}$ is quite different in the analyses of the Daya Bay evolution data and the reactor rates. In the first analysis $r_{235}$ and $r_{239}$ are correlated, whereas in the second analysis they are slightly anticorrelated. Moreover, the analysis of the reactor rates prefers a larger value of $r_{235}$ and a smaller value of $r_{239}$ than the analysis of the Daya Bay evolution data. The results of the analysis of the reactor rates are

$$
\begin{aligned}
& r_{235}=0.950 \pm 0.013, \\
& r_{239}=0.873 \pm 0.064,
\end{aligned}
$$

which imply

$$
\begin{aligned}
& \sigma_{f, 235}=6.36 \pm 0.09 \\
& \sigma_{f, 239}=3.84 \pm 0.28
\end{aligned}
$$

Figure 4 show the allowed region in the $\sin ^{2} 2 \vartheta_{e e}-\Delta m_{41}^{2}$ plane obtained from the fit of the reactor rates in the OSC model. One can see that there in only one region allowed at $1 \sigma$ around the best-fit point given in table 2 , but the $2 \sigma$ allowed regions do not have an upper bound for $\Delta m_{41}^{2}$. The $3 \sigma$ allowed region does not have a lower bound for $\sin ^{2} 2 \vartheta_{e e}$, because oscillations are favored over the no-oscillation case only at the $2.7 \sigma$ level.

From a comparison of figures 4,5 , and 6 one can see that the variations of $r_{235}$ and $r_{239}$ in the 235+OSC and 239+OSC models, respectively, have small effects on the allowed region in the $\sin ^{2} 2 \vartheta_{e e}-\Delta m_{41}^{2}$ plane, in agreement with the best-fit values close to one of $r_{235}$ and $r_{239}$ in table 2 .

\section{Combined analysis}

In this section we present the results of the combined fits of the reactor rates in table 1 of ref. [12] (without the 2016 Daya Bay rate) and the 2017 Daya Bay evolution data [11].

The results of the fits with the models described in section 1 are listed in table 3 .

From table 3 one can see that all the models have an excellent goodness-of-fit. The OSC model has a better goodness-of-fit than the 235 model. There is little improvement of the goodness-of-fit from the 235 model to the $235+239$ model, whereas the goodness-of-fit improves significantly in the $235+$ OSC model and especially in the $239+$ OSC model. 


\begin{tabular}{|cccccc|}
\hline & 235 & $235+239$ & OSC & $235+$ OSC & $239+$ OSC \\
\hline$\chi_{\min }^{2}$ & 25.3 & 24.8 & 23.0 & 20.2 & 17.5 \\
$\mathrm{NDF}$ & 32 & 31 & 31 & 30 & 30 \\
$\mathrm{GoF}$ & $79 \%$ & $78 \%$ & $85 \%$ & $91 \%$ & $100 \%$ \\
$\Delta m_{41}^{2}$ & - & - & 0.48 & 0.48 & 0.48 \\
$\sin ^{2} 2 \vartheta_{e e}$ & - & - & 0.14 & 0.11 & 0.15 \\
$r_{235}$ & 0.934 & 0.934 & - & 0.987 & - \\
$r_{239}$ & - & 0.970 & - & - & 1.099 \\
\hline
\end{tabular}

Table 3. Fits of the reactor rates in table 1 of ref. [12] (without the 2016 Daya Bay rate) and the 2017 Daya Bay evolution data [11].

The comparison of the nested models 235 and $235+\mathrm{OSC}$ give $\Delta \chi^{2}=5.1$ with two degrees of freedom. Hence, the $p$-value of the null hypothesis 235 is $7.8 \%$ and it can be rejected in favor of the introduction of active-sterile neutrino oscillations only at $1.8 \sigma$. As a check, with a Monte Carlo simulation we obtained a $p$-value of $5.1 \%$, which corresponds to $1.9 \sigma$.

The 235 and $235+239$ models have $\Delta \chi_{\min }^{2}=2.3$ and 1.8 with respect to the OSC model and our Monte Carlo comparison disfavors them at $1.7 \sigma$ and $2.2 \sigma$, respectively.

The 235, 235+239, OSC, and 235+OSC models have $\Delta \chi_{\min }^{2}=7.8,7.3,5.5$, and 2.7 with respect to the $239+\mathrm{OSC}$ model and our Monte Carlo comparison disfavors them at $4.2 \sigma, 2.9 \sigma, 2.4 \sigma$, and $3.5 \sigma$, respectively.

From figure 2 one can see that in the 235 model the combined fit indicates a value of $r_{235}$ intermediate between those obtained from the analyzes of the Daya Bay evolution data and the reactor rates. The result is

$$
r_{235}=0.934 \pm 0.010
$$

which gives

$$
\sigma_{f, 235}=6.25 \pm 0.07 \text {. }
$$

This is a determination of $\sigma_{f, 235}$ with smaller uncertainty than that obtained in eq. (2.7) from the Daya Bay evolution data and that obtained in eq. (3.2) from the reactor rates.

Figure 3 shows that in the case of the $235+239$ model the determination of $r_{235}$ and $r_{239}$ from the combined fit improves the uncertainties of the two parameters with respect to those obtained from the separate analyses of the Daya Bay evolution data and the reactor rates The results are

$$
\begin{aligned}
& r_{235}=0.934 \pm 0.009, \\
& r_{239}=0.970 \pm 0.032,
\end{aligned}
$$

which give

$$
\begin{aligned}
& \sigma_{f, 235}=6.25 \pm 0.06, \\
& \sigma_{f, 239}=4.27 \pm 0.14 .
\end{aligned}
$$


Within the uncertainties, these results are compatible with those obtained in ref. [30] with different assumptions on the uncertainties of $\sigma_{f, 238}$ and $\sigma_{f, 241}$. Note that here we performed a full analysis of the Daya Bay evolution data using the complete information available in the Supplemental Material of ref. [11] whereas in ref. [30] the Daya Bay evolution data have been taken into account with a Gaussian approximation of the $\chi^{2}$ distribution in figure 3 of ref. [11].

Figure 4 show the allowed region in the $\sin ^{2} 2 \vartheta_{e e}-\Delta m_{41}^{2}$ plane in the OSC model. The allowed regions are smaller than those obtained from the fit of the reactor rates and there is a $3 \sigma$ lower bound for $\sin ^{2} 2 \vartheta_{e e}$, because oscillations are favored over the no-oscillation case at the $3.1 \sigma$ level. However, there is no upper bound for $\Delta m_{41}^{2}$ at $2 \sigma$, because at that confidence level the data can be fitted with an averaged oscillation probability which does not depend on the source-detector distance.

Comparing figures 4 and 5 , one can see that the variation of $r_{235}$ in the $235+\mathrm{OSC}$ enlarges the allowed regions towards lower values of $\sin ^{2} 2 \vartheta_{e e}$ and there is no lower bound for $\sin ^{2} 2 \vartheta_{e e}$ at $2 \sigma$, because oscillations are favored over the no-oscillation case only at $1.4 \sigma$. This is due to the preference for values of $r_{235}$ smaller than one, as shown by the best-fit value in table 3.

Figure 6 shows that the best-fitting model 239+OSC gives the strongest indication in favor of oscillations, which are favored over the no-oscillation case at 3.0 $\sigma$. This is due to the preference for values of $r_{239}$ larger than one, as shown by the best-fit value in table 3 .

\section{Conclusions}

In this paper we analyzed the Daya Bay evolution data [11] in the 235, 235+239, OSC, $235+$ OSC, and 239+OSC models described in section 1, which allow to compare the fits of the data under the hypotheses of variations of the ${ }^{235} \mathrm{U}$ and ${ }^{239} \mathrm{Pu}$ reactor antineutrino fluxes with respect to the Saclay+Huber theoretical value $[4,5,9]$ and short-baseline activesterile neutrino oscillations, taking into account the theoretical uncertainties of the reactor antineutrino fluxes. We found that the best explanation of the Daya Bay evolution data is the 235 model with a variation of the ${ }^{235} \mathrm{U}$ flux with respect to the Saclay+Huber theoretical value $[4,5,9]$. Comparing the OSC model of active-sterile neutrino oscillations with the 235 model, we found that it is disfavored at $2.6 \sigma$.

We also compared the OSC model with the $235+239$ model which allows independent variations of the ${ }^{235} \mathrm{U}$ and ${ }^{239} \mathrm{Pu}$ fluxes with respect to Saclay+Huber theoretical values $[4,5,9]$. We found that the OSC model is disfavored at $2.5 \sigma$. This result is slightly less stringent than the $2.8 \sigma$ obtained by the Daya Bay collaboration [11] without considering the theoretical uncertainties.

The Daya Bay evolution data can also be fitted well with the $235+$ OSC model, with a suppression of the ${ }^{235} \mathrm{U}$ flux and neutrino oscillations, or with the $239+$ OSC model, with an enhancement of the ${ }^{239} \mathrm{Pu}$ flux and relatively large neutrino oscillations.

We also performed a similar analysis of the reactor antineutrino data which were available before the release of the Daya Bay fuel evolution data in ref. [11]. In this case, we found that the best explanation of the data is the OSC model with active-sterile neutrino 
oscillations, which depend on the source-detector distance and fit the rates measured by reactor experiments with a source-detector distance between about 10 and $100 \mathrm{~m}$ better than the distance-independent suppression of the reactor antineutrino flux given by suppressions of the ${ }^{235} \mathrm{U}$ and ${ }^{239} \mathrm{Pu}$ fluxes. In this case, the 235 model with a suppression of the ${ }^{235} \mathrm{U}$ flux only is disfavored at $3.1 \sigma$ and the $235+239$ model with independent suppressions of the ${ }^{235} \mathrm{U}$ and ${ }^{239} \mathrm{Pu}$ fluxes is disfavored at $2.8 \sigma$. As with the fit of the Daya Bay evolution data, composite models including both variations of the ${ }^{235} \mathrm{U}$ or ${ }^{239} \mathrm{Pu}$ fluxes and active-sterile oscillations provide good fits to the global reactor rate data.

Finally, we performed combined fits of the Daya Bay evolution data and the other reactor rates and we found that all the considered models fit well the data. The OSC model has a better goodness-of-fit than the 235 and $235+239$ models, which are almost equivalent. We obtained better fits of the data with the composite 235+OSC and 239+OSC models. In particular, the best-fit model is $239+\mathrm{OSC}$, with an increase of the ${ }^{239} \mathrm{Pu}$ flux with respect to the Saclay+Huber theoretical value $[4,5,9]$ and relatively large activesterile neutrino oscillations.

In conclusion, although the recent Daya Bay evolution data [11] disfavor short-baseline active-sterile neutrino oscillations over a suppression of the ${ }^{235} \mathrm{U}$ reactor antineutrino flux or independent suppressions of the ${ }^{235} \mathrm{U}$ and ${ }^{239} \mathrm{Pu}$ fluxes, the result is reversed in the analysis of the other available reactor antineutrino data. Both sets of data are individually well-fitted by composite models with variations of the ${ }^{235} \mathrm{U}$ or ${ }^{239} \mathrm{Pu}$ fluxes and activesterile neutrino oscillations. The combined data set indicates a preference for the composite models and, in particular, the best fit is obtained with the 239+OSC model, through an enhancement of the ${ }^{239} \mathrm{Pu}$ flux and relatively large oscillations. However, while these combined fits suggest a preference for models including sterile neutrinos, the significant uncertainties in the reactor rate measurements and the high goodness-of-fits observed for models both with and without sterile neutrinos make it clear that the search for the explanation of the reactor antineutrino anomaly [9] still remains open. We hope that it will be solved soon by the new short-baseline reactor neutrino experiments which will measure the reactor antineutrino flux from reactors with different fuel compositions: highly enriched ${ }^{235} \mathrm{U}$ research reactors for PROSPECT [31], SoLid [32], and STEREO [33], and commercial reactors with mixed fuel compositions for DANSS [34] and Neutrino-4 [35].

\section{Acknowledgments}

We would like to thank the Daya Bay collaboration for useful discussions and information on the Daya Bay evolution data. The work of X.P. Ji was supported by the National Natural Science Foundation of China (Grants No. 11235006 and No. 11475093) and by the CAS Center for Excellence in Particle Physics (CCEPP). The work of Y.F. Li was supported in part by the National Natural Science Foundation of China under Grant Nos. 11305193 and 11135009, by the Strategic Priority Research Program of the Chinese Academy of Sciences under Grant No. XDA10010100, and by CCEPP. The work of B.R. Littlejohn was partially supported by the DOE Office of Science, under award No. DE-SC0008347. 
Open Access. This article is distributed under the terms of the Creative Commons Attribution License (CC-BY 4.0), which permits any use, distribution and reproduction in any medium, provided the original author(s) and source are credited.

\section{References}

[1] C. Bemporad, G. Gratta and P. Vogel, Reactor-based neutrino oscillation experiments, Rev. Mod. Phys. 74 (2002) 297 [hep-ph/0107277].

[2] S.-B. Kim, T. Lasserre and Y. Wang, Reactor Neutrinos, Adv.High Energy Phys. 2013 (2013) 453816.

[3] A.C. Hayes and P. Vogel, Reactor Neutrino Spectra, Ann. Rev. Nucl. Part. Sci. 66 (2016) 219 [arXiv: 1605.02047].

[4] T.A. Mueller et al., Improved predictions of reactor antineutrino spectra, Phys. Rev. C $\mathbf{8 3}$ (2011) 054615 [arXiv:1101.2663].

[5] P. Huber, Determination of antineutrino spectra from nuclear reactors, Phys. Rev. C 84 (2011) 024617 [arXiv:1106.0687].

[6] P. Vogel, G.K. Schenter, F.M. Mann and R.E. Schenter, Reactor Anti-neutrino Spectra and Their Application to Anti-neutrino Induced Reactions. 2., Phys. Rev. C 24 (1981) 1543 [INSPIRE].

[7] K. Schreckenbach, G. Colvin, W. Gelletly and F. Von Feilitzsch, Determination of the anti-neutrino spectrum from U-235 thermal neutron fission products up to 9.5-MeV, Phys. Lett. B 160 (1985) 325 [INSPIRE].

[8] A.A. Hahn, K. Schreckenbach, G. Colvin, B. Krusche, W. Gelletly and F. Von Feilitzsch, Anti-neutrino Spectra From ${ }^{241} \mathrm{Pu}$ and ${ }^{239} \mathrm{Pu}$ Thermal Neutron Fission Products, Phys. Lett. B 218 (1989) 365 [INSPIRE].

[9] G. Mention et al., The Reactor Antineutrino Anomaly, Phys. Rev. D 83 (2011) 073006 [arXiv: 1101.2755] [INSPIRE].

[10] S. Gariazzo, C. Giunti, M. Laveder, Y.F. Li and E.M. Zavanin, Light sterile neutrinos, J. Phys. G 43 (2016) 033001 [arXiv: 1507.08204] [InSPIRE].

[11] DaYA BAY collaboration, F.P. An et al., Evolution of the Reactor Antineutrino Flux and Spectrum at Daya Bay, Phys. Rev. Lett. 118 (2017) 251801 [arXiv: 1704.01082] [InSPIRE].

[12] S. Gariazzo, C. Giunti, M. Laveder and Y.F. Li, Updated Global 3+1 Analysis of Short-BaseLine Neutrino Oscillations, JHEP 06 (2017) 135 [arXiv:1703.00860] [INSPIRE].

[13] Y. Declais et al., Study of reactor anti-neutrino interaction with proton at Bugey nuclear power plant, Phys. Lett. B 338 (1994) 383 [INSPIRE].

[14] A.A. Kuvshinnikov, L.A. Mikaelyan, S.V. Nikolaev, M.D. Skorokhvatov and A.V. Etenko, Measuring the anti-electron-neutrino $+p \rightarrow n+e+$ cross-section and beta decay axial constant in a new experiment at Rovno NPP reactor (in Russian), JETP Lett. 54 (1991) 253 [INSPIRE].

[15] Y. Declais et al., Search for neutrino oscillations at 15-meters, 40-meters and 95-meters from a nuclear power reactor at Bugey, Nucl. Phys. B 434 (1995) 503 [INSPIRE].

[16] CALTECH-SIN-TUM collaboration, G. Zacek et al., Neutrino Oscillation Experiments at the Gosgen Nuclear Power Reactor, Phys. Rev. D 34 (1986) 2621 [INSPIRE]. 
[17] H. Kwon et al., Search for Neutrino Oscillations at a Fission Reactor, Phys. Rev. D 24 (1981) 1097 [InSPIRE].

[18] A. Hoummada, S. Lazrak Mikou, G. Bagieu, J. Cavaignac and D. Holm Koang, Neutrino oscillations I.L.L. experiment reanalysis, Appl. Radiat. Isot. 46 (1995) 449.

[19] G.S. Vidyakin et al., Detection of Anti-neutrinos in the Flux From Two Reactors, Sov. Phys. JETP 66 (1987) 243 [INSPIRE].

[20] G.S. Vidyakin et al., Bounds on the neutrino oscillation parameters for reactor anti-neutrinos, Sov. Phys. JETP 71 (1990) 424 [INSPIRE].

[21] G.S. Vidyakin et al., Limitations on the characteristics of neutrino oscillations, JETP Lett. 59 (1994) 390 [INSPIRE].

[22] A.I. Afonin, S.N. Ketov, V.I. Kopeikin, L.A. Mikaelyan, M.D. Skorokhvatov and S.V. Tolokonnikov, A Study of the Reaction $\bar{\nu}_{e}+P \rightarrow e^{+}+N$ on a Nuclear Reactor, Sov. Phys. JETP 67 (1988) 213 [INSPIRE].

[23] Z.D. Greenwood et al., Results of a two position reactor neutrino oscillation experiment, Phys. Rev. D 53 (1996) 6054 [INSPIRE].

[24] NUCIFER collaboration, G. Boireau et al., Online Monitoring of the Osiris Reactor with the Nucifer Neutrino Detector, Phys. Rev. D 93 (2016) 112006 [arXiv:1509.05610] [INSPIRE].

[25] CHOOZ collaboration, M. Apollonio et al., Search for neutrino oscillations on a long baseline at the CHOOZ nuclear power station, Eur. Phys. J. C 27 (2003) 331 [hep-ex/0301017] [INSPIRE].

[26] F. Boehm et al., Final results from the Palo Verde neutrino oscillation experiment, Phys. Rev. D 64 (2001) 112001 [hep-ex/0107009] [INSPIRE].

[27] DaYa Bay collaboration, F.P. An et al., Improved Measurement of the Reactor Antineutrino Flux and Spectrum at Daya Bay, Chin. Phys. C 41 (2017) 013002 [arXiv:1607.05378] [INSPIRE].

[28] H. Seo, Recent Results from RENO, talk presented at AAP 2016, Applied Antineutrino Physics, Liverpool, U.K., 1-2 December 2016.

[29] Double Chooz collaboration, private communication.

[30] C. Giunti, Improved Determination of the ${ }^{235} \mathrm{U}$ and ${ }^{239} \mathrm{Pu}$ Reactor Antineutrino Cross sections per Fission, Phys. Rev. D 96 (2017) 033005 [arXiv:1704.02276] [InSPIRE].

[31] PROSPECT collaboration, J. Ashenfelter et al., The PROSPECT Physics Program, J. Phys. G 43 (2016) 113001 [arXiv:1512.02202] [INSPIRE].

[32] SoLid collaboration, N. Ryder, First results of the deployment of a SoLid detector module at the SCK-CEN BR2 reactor, PoS(EPS-HEP2015) 071 [arXiv:1510.07835] [INSPIRE].

[33] STEREO collaboration, V. Hélaine, Sterile neutrino search at the ILL nuclear reactor: the STEREO experiment, arXiv: 1604.08877 [INSPIRE].

[34] I. Alekseev et al., DANSS: Detector of the reactor AntiNeutrino based on Solid Scintillator, 2016 JINST 11 P11011 [arXiv:1606.02896] [INSPIRE].

[35] A. Serebrov et al., Experiment Neutrino-4 Search for Sterile Neutrino with Multisection Detector Model, PoS(INPC2016) 255 [arXiv: 1702.00941] [INSPIRE]. 